\title{
Essais
}

Revue interdisciplinaire d'Humanités

14 | 2018

Plurilinguismes en construction

\section{Vieux démons, nouveaux défis dans l'enseignement des langues}

Vers une didactique réticulaire

Pierre Martinez

\section{(2) OpenEdition}

\section{Journals}

Édition électronique

URL : http://journals.openedition.org/essais/372

DOI : 10.4000/essais.372

ISSN : 2276-0970

\section{Éditeur}

École doctorale Montaigne Humanités

Édition imprimée

Date de publication : 1 juin 2018

Pagination : 157-177

ISBN : 979-10-97024-06-2

ISSN : $2417-4211$

\section{Référence électronique}

Pierre Martinez, «Vieux démons, nouveaux défis dans l'enseignement des langues », Essais [En ligne],

14 | 2018, mis en ligne le 01 décembre 2019, consulté le 10 décembre 2020. URL : http://

journals.openedition.org/essais/372 ; DOI : https://doi.org/10.4000/essais.372 


\title{
Vieux démons, nouveaux défis dans l'enseignement des langues Vers une didactique réticulaire
}

\section{Pierre Martinez}

\author{
Langues vivantes : \\ Les malheurs de la France viennent de ce quion n'en \\ sait pas assez. \\ Innovation : \\ Toujours dangereuse. \\ G. Flaubert, Dictionnaire des idées reçues.
}

À l'origine, l'idée de donner ce texte à une revue scientifique de la qualité des Essais, à l'Université Bordeaux Montaigne, m’a paru difficile à concrétiser. Deux raisons à cela. D'une part, la conférence qui avait été à l'origine de la proposition relevait d'un genre oral à l'évidence spécifique et le passage à l'écrit en laisserait voir bien des imperfections et des insuffisances. Ainsi, des questions abordées lors de cette intervention à l'Université Bordeaux Montaigne avaient donné lieu à des échanges qui ne figureraient pas ici. D'autre part, beaucoup de thèmes abordés (la médiation, le contexte, l'historicité...) demandaient à être creusés et cet approfondissement ne peut guère trouver sa place que dans des textes plus longs et plus étayés, qui sont d'ailleurs, pour ce qui me concerne, en voie de publication. ${ }^{1}$

Le projet même sur lequel nous nous étions mis d'accord, les organisateurs et moi-même, tenait en revanche toujours aussi solidement. ${ }^{2} \mathrm{Ni}$ dans la

1 Pierre Martinez, La Didactique des Langues Étrangères, Paris, PUF, 1996. Une $8^{\mathrm{e}}$ édition de cet opuscule, fortement remanié dans ses orientations scientifiques, est parue en novembre 2017, ainsi que, début 2018, un ouvrage intitulé Un regard sur l'enseignement des langues. Des sciences du langage aux NBIC, Paris, Éditions des Archives Contemporaines. Le titre lui-même dit assez quel déplacement épistémologique est opéré dans ce dernier volume : le concept de didactique réticulaire, esquissé dans le présent article, y est, bien entendu, plus largement explicité.

2 Qu'il me soit permis de remercier particulièrement la Professeure Mariella Causa, responsable au plus haut niveau de ce qui peut faire avancer la connaissance du champ didactique à l'Université Bordeaux Montaigne et le Professeur Sandro Landi, directeur de l'École Doctorale Montaigne-Humanités. 
conférence, ni dans le présent article, l'objet de la réflexion ne serait de retracer l'archéologie de la didactique, ce qui a déjà été fait dans de bons ouvrages. ${ }^{3}$ Il ne serait pas non plus, bien entendu, de se livrer à un exercice autobiographique tel qu'un universitaire émérite (encore directeur de thèses, mais disons, étymologiquement, sorti des cadres de l'enseignement) pourrait en concevoir avec gourmandise l'intention égocentrique.

Le propos est à la fois plus simple et plus ambitieux. Il consiste à dégager et mettre en lumière, dans ses grandes orientations, la problématique actuelle de la didactique des langues étrangères et secondes (désormais DLES), un domaine scientifique qui vise à faciliter l'appropriation des langues et à donner aux acteurs de ce domaine les moyens d'optimiser cette appropriation.

Pour cela, il m'a semblé judicieux d'associer deux parcours. Il s'agit, d'un côté, de dessiner l'itinéraire du professionnel que j'ai été pendant longtemps, immergé ou plutôt "embarqué " (Pascal rejoint Montaigne), vivant les vicissitudes d'un champ théorico-pratique, ancré dans la formation humaine et professionnelle, dans l'intervention sociale. De l'autre, c'est bien de ces vicissitudes qu'on voudrait retracer quelques temps forts, et la didactique des langues en a été, dans les quatre ou cinq dernières décennies, riche en exemples : des méthodologies traditionnelles, fondées sur la grammaire, le texte littéraire et la traduction interlinguale, jusquaux approches communicatives et actionnelles qui ont fait pencher le balancier vers d'autres zones d'attente, on peut repérer bien des évolutions, des contradictions, des avancées aussi. Une partie de cette dynamique tient au fait suivant : si la DLES peut être considérée, à certains égards, comme une discipline, que ce soit en fonction de sa désignation académique ou parce qu' elle constitue bien une communauté de pratiques de recherche, elle est davantage à mes yeux un domaine scientifique qui définit son identité par la diversité des polarités vers lesquelles il s'est tourné et par les ressources auxquelles il a recours. Certes, ce sont les sciences du langage qui viennent aussitôt à l'esprit, mais bien d'autres aussi, de l'ethnographie de la communication et des sciences sociales en général, jusqu'aux technosciences numériques et cognitives de notre temps, et j'insisterai donc fortement sur cette diversité.

La DLES a été depuis plusieurs décennies en voie de construction, de durcification (comme on dit aux Antilles), de solidification. Elle est à la recherche de son équilibre, ou plutôt de son centre de gravité, et elle continue de le faire, justement parce qu' elle est vivante et qu' elle n' existe qu'en situation. Il m'apparait vain - j'en montrerai dans ce qui suit immédiatement quelques raisons - d'essayer de parler de la didactique sans tenir compte du contexte dans lequel elle est opératoire. Une didactique hors du contexte, décontextua-

3 Par exemple : Christian Puren, Histoire des méthodologies de l'enseignement des langues, Paris, Nathan-CLE international, 1988. Claude Germain, Evolution de l'enseignement des langues: 5000 ans d'histoire, Paris, CLE international, 1993. 
lisée (ou, pis encore, atopique, sans contexte) ne fait pas sens. C'est l'ornithologie sans l'air et sans la pesanteur. On le comprendra mieux par la suite, mais les exemples que l'expérience de terrain peut en fournir ne manquent pas, à commencer par celui que donnent, à l'heure actuelle, l'Union Européenne et son texte-phare (pour ne pas dire son mantra), le CECRL. ${ }^{4}$

Le discours de la DLES reste donc en voie de formation, il n'est ni figé sur une théorie (fût-elle le nec plus ultra actuel que semble être l'approche actionnelle), ni passéiste en ce qu'il regretterait la perte de ses fondamentaux anciens, notamment la relation historique du pédagogue, du maître (magister) à son élève (discipulus). Il me resterait à expliquer le choix de mon titre, mais peut-être les mots "vieux démons, nouveaux défis " parleront-ils tout seuls au long des pages : promesses et dangers, pour et contre, avant et après, contradictions, questions non résolues, difficultés en vue.

Pour en venir à l'organisation de mon propos, j'adopterai très simplement quatre angles d'approche. Ils correspondent au trajet personnel que j'ai mis en regard de l'évolution du domaine de la DLES. ${ }^{5}$ A travers quatre types d'expériences, longues et prenantes, sur le plan des savoirs et, plus encore, des contacts et des enrichissements culturels et scientifiques, j'entends ainsi donner l'idée de ce que j'ai pu vivre sur quatre continents, comme enseignant, chercheur et formateur d'enseignants. Si la diversité des contextes conditionne l'intervention, il m’appartiendra de mettre en évidence, de donner à sentir, à partir d'une série d'exemples regroupés thématiquement, comment la didactique s'attache non pas à commander l'humain, mais à organiser les choses. ${ }^{6}$

Il me semble que le dégagement de la problématique dont j’ai parlé plus haut sera peut-être utile aux jeunes chercheurs, en master ou en thèse, en ce qu'il illustre une posture ou une position de recherche qui réunit expertise (toujours imparfaite) et expériences (toujours à poursuivre). À l'instar de son objet, le discours tenu dans ce texte, loin de trop grandes certitudes, reste toujours en évolution, ouvert à un horizon d'attente mais certainement pas à une quelconque doxa. Il sera, je l'espère en tout cas, une occasion d'approfondir leur réflexion pour ceux qui s'engageront dans cette voie et dans ce métier (au sens de ministerium, autant une vocation qu'une fonction) aux facettes variées, leur faisant se souvenir toujours des mots de Dante Alighieri, qui n'aimait pas moins le doute que les certitudes. ${ }^{7}$

4 CECRL : Cadre Européen Commun de Référence pour les langues, Conseil de l'Europe, Division des Politiques Linguistiques, Strasbourg, 2001.

5 La linéarité chronologique est évidemment parfois mise de côté au profit du regroupement thématique.

6 Pierre Martinez, "Contextualiser, comparer, relativiser : jusqu'où aller ? ", in Philippe Blanchet et Patrick Chardenet (éds), Guide de recherche en didactique des langues et des cultures : une approche contextualisée, Paris, EAC, édition 2011, p. 509-517.

7 "Che non men che saper, dubbiar m'aggrada.", Enfer, XI. 
Une permanence des questions s'impose donc. J'en retiens trois. D'abord, la question théorique, qui pousse à constater l'extension épistémologique d'un domaine dont les objets, les processus et les méthodes sont en constante redéfinition au fil des mutations. Ainsi, il y a la DLES avant et après l'apparition du numérique, qui fait que l'apprenant n'est plus tout à fait le même en face-à-face et dans un dispositif collaboratif; il y a les interrogations qu'on éprouvera au vu de sa diversité : il s'agit aussi de savoir par quel bout prendre les connaissances, les phénomènes, les opérations, avec le sentiment qu'on a toujours plus d'information mais toujours moins de compréhension ; il y a, enfin, les problèmes que pose sa transmission, dans la médiation, dans la formation. En somme, la grande quantité des variables relatives à l'extension, à la diversité, à la transmission (même si cette quantité de variables est certes assez commune dans les sciences qui touchent à l'humain) donne bien l'impression d'appréhender - métaphore certes assez policière - les contours d'un domaine dont la complexité se refuse à l'analyse.

Or le mouvement de complexification s'est accéléré brutalement avec de récentes transformations qui ne sont pas que de surface, et une offre méthodologique élargie renouvelle ces thèmes de réflexion ou en impose de nouveaux, naguère insoupçonnés. Ainsi en est-il des interactions dans le processus de transmission des savoirs et de leur traitement quand il fait intervenir à la fois le formateur, l'apprenant, la machine, le réseau social (qui fait quoi à quel moment et pourquoi ?). Ainsi en-est-il également de l'évaluation des performances en langue étrangère quand on passe de la copie, de la dissertation ou du Questionnaire à Choix multiple (QCM) à des productions qui font intervenir des dispositifs autonomes ou informels, fondés sur la mobilité, la collaboration ou la pédagogie de projet (comment évaluer un stage ou un travail de groupe ?). ${ }^{8}$

\section{Penser la DLES...}

Avant tout, peut-être convient-il de préciser ce qu'on entend dans ces lignes par langue étrangère et langue seconde, car cette démarcation est de celles qui amènent, au premier chef, à distinguer des contextes, des interventions et des théorisations différentes et contribuent à la complexité évoquée ci-dessus. Le lecteur bien informé de ces questions me pardonnera cette précaution.

Il faut partir de l'ancrage social des termes pour bien comprendre leur signification et leurs effets sur le répertoire linguistique des individus et les contextes d'apprentissage évoqués plus loin dans cet article. Classiquement, on désigne comme langue première celle qui a été acquise chronologique-

8 J'ose à peine mentionner le rôle que pourraient prendre les métadonnées (analyse du processus d'apprentissage et des conditions de production de la performance) et les mégadonnées ( $b i g$ data " autour de l'apprenant) dans l'évaluation actuelle d'une performance langagière. 
ment avec le développement du langage. Comme, dans un certain nombre de sociétés, l'éducation est le fait de l'environnement maternel, on parle communément de langue maternelle pour désigner la langue première. Une simple observation montre quelle valeur psychoaffective acquiert pour chacun cette langue maternelle, quelle part constitutive de l'identité et de la personne elle recouvre. Toute langue acquise ou apprise sera à la suite qualifiée de seconde. Cependant, langue seconde a pris une acception spécifique, quand le terme est employé collectivement et c'est le cas, notamment pour le français, dans des conditions historiques précises.

D'une part, il est arrivé qu'un des parlers en usage se soit vu conférer par un pouvoir dominant une place particulière dans le répertoire verbal, une légitimité et un pouvoir symbolique, ${ }^{9}$ avec un environnement favorable à son apprentissage et à son utilisation, comme ce fut ou est encore le cas dans les situations coloniales et postcoloniales des gouvernorats, ceux des Antilles, de l'Océan Indien, du Maghreb, de l'Afrique subsaharienne ou de l'ancienne Indochine, ou du raj britannique en Inde, etc. On a pu parler d'une " logistique » de la langue seconde (souvent promue langue officielle dans l'ensemble des langues locales ou nationales en usage), qui fait référence à des emplois administratifs, commerciaux, médiatiques et, bien sûr scolaires.

D'autre part, on connaît en France même, un français langue seconde désignant le statut que revêt la langue d'accueil pour des populations immigrées temporairement ou non, migrants, réfugiés ou étudiants, dans la mesure où, là encore, les conditions de l'apprentissage et de l'emploi sont affectées par des situations relationnelles ou institutionnelles particulières. ${ }^{10}$

Maintenant, penser la DLES, face à cette complexité dont mon préambule a voulu avertir le lecteur, cela va ressembler à ce qu'a dû ressentir Montaigne en rédigeant son chapitre des Essais intitulé les Cannibales. La question qui va hanter la modernité, celle de la Différence, amène, on s'en souvient, Montaigne à penser l'homme que l'on prétend sauvage, "le Sauvage " qui arrivait sur les quais de Bordeaux, non selon des catégories reçues (le canon du civilisé et du barbare), mais autrement : en termes d'originalité et simultanément de spécificité et d'humanité. C'est en ces termes d'une découverte sans préjugés que je voudrais traiter des points forts et des préoccupations qui peuvent retenir l'attention en DLES.

Les quatre groupes de contextes présentés permettent d'adopter des angles d'approche consécutifs et différents. Ce processus fait apparaître des polarités, liées à des zones scientifiques et culturelles. Il n'y a rien d'inquiétant à ce que

9 Référence, bien entendu, à l'ouvrage fondateur de Pierre Bourdieu, Ce que parler veut dire. Économie des échanges linguistiques, Paris, Fayard, 1982.

10 Sur la notion de langue seconde : Jean-Pierre Cuq, Le français langue seconde. Origine d'une notion et implications didactiques, Paris, Hachette, 1991. Pierre Martinez (éd.), Français langue seconde : apprentissage et curriculum, Paris, Maisonneuve et Larose, 2003. 
se construisent deux images qui finissent par se confondre. Avec l'humilité qui convient, c'est ainsi que ma représentation de l'évolution de tout un domaine scientifique a évolué avec ce qui pouvait m’apparaître, de New York à Pointeà-Pitre, d'Alger à Hanoï, de Paris 8 à Djibouti, acteur - parmi d'autres - de ce domaine.

Par commodité, j'énumère ces ensembles qui seront, successivement :

1) L'Occident, entendu comme intégrant l'Europe et l'Amérique du Nord et des zones périphériques pour lesquelles l'analogie est possible.

2) Les mondes créoles, limités en l'occurrence, à mon expérience aux Antilles, à la Réunion, à l'Ile Maurice et à Haïti.

3) L'Afrique, avec le Maghreb, l'Éthiopie, Djibouti et l'Afrique de l'Ouest.

4) Enfin, une partie de l'Asie, celles du Sud-Est et du Nord-Est surtout, comprenant l'Inde, la Corée, le Japon et le Vietnam.

Au delà des simplifications qu'impliquent ces regroupements (les pays du Maghreb offrent beaucoup de similitudes mais aussi de différences), la réflexion portera sur ce qu'ils ont pu m'aider à comprendre après coup, bien sûr, dans quatre secteurs essentiels, étant entendu que des questions tout aussi importantes ont dû être mises ici de côté :

1) La conceptualisation d'une épistémologie (l'épistémè est, on le sait, le processus de fabrication, de construction du savoir d'un domaine : objets, méthodes, résultats.)

2) L'au-delà des frontières disciplinaires, ensuite, qui autorise à étendre l'empan de l'observation et à opérer un dessillement du regard, comme dit Péguy.

3) Dans un troisième temps, les problèmes d'ingénierie éducative de terrain, sur lesquels j'ai eu particulièrement l'occasion de travailler autour de projets de développements économiques et culturels internationaux.

4) Enfin, autour de la notion de "contexte", même si le mot met mal en valeur sa richesse sémantique, trois types de questions s'agrègent : le géopolitique, le cognitif notamment dans sa dimension ethnologique, le numérique. J’y reviendrai plus loin.

\section{OCCIDENT. La tentation épistémologique}

La DLES s'est constituée épistémologiquement comme une praxéologie, c'est-à-dire qu'elle englobe un logos (un discours raisonné) et une praxis (un agir social). Elle fonctionne comme un dispositif de production, de justification et d'intelligibilité des techniques de formation, ainsi que l'avait montré plus tôt Chevallard pour les mathématiques. Conduisant à des conclusions opératoires, elle contribue à transformer les pratiques des acteurs et, en retour, s'enrichit des observations qu'elle fait de ces pratiques. Dans les années 1960- 
1970, deux expériences consécutives me permettent, d'abord en formation au Centre de Linguistique Appliquée de Besançon, puis comme lecteur aux ÉtatsUnis (université de l'État de New York, New Paltz), de connaître ce qui était encore la linguistique appliquée, et plus tard, de m'initier à une ethnographie de la communication dont on sait quelle influence elle eut sur les méthodes et les approches communicatives en enseignement des langues.

\section{Le CLA de Besançon, New Paltz (NY) et les fondamentaux}

Le CLAB était alors, avec quelques autres institutions en France, dont le renommé Crédif rattaché à l'ENS de Saint-Cloud, ${ }^{11}$ l'un des meilleurs lieux d'exploration et de recherche qu'on pût imaginer pour l'enseignement des langues. Fondé par un lexicologue, Quemada, à la fin des années 60, le CLAB affichait ses liens avec ce développement de la linguistique dite "appliquée " dont Suso Lopez écrit, citant Puren, 1988 : "Quant à l'université française, elle reste en général hostile ou étrangère à ce mouvement, et c'est dans une petite université de province que Bernard Quemada fonde en 1961 le troisième grand organisme de ce type $\|^{12}$ On implique et on applique donc la linguistique au CLAB. On y réfléchit avec S. Moirand, R. Porquier et J. Montredon, sur des données de départ qui sont celles des méthodes structuro-globales audiovisuelles, dans une étroite subordination à la linguistique distributionnelle et à la psychologie béhavioriste : correction des erreurs, laboratoire de langue, dialogue en situation, exercice structural, film fixe sont les ingrédients de cette méthodologie qui se rattache historiquement aux apprentissages rapides destinés, pendant la seconde guerre mondiale, aux militaires américains des opérations extérieures (ASTP). Peu de réalité sociale, sinon bien convenue, alors, au fil des leçons. Quand nous travaillons sur la méthode C'est le Printemps, en 1972, voilà qu'apparaît une image hors du commun de la société française : un auto-stoppeur quittant sa Bretagne natale pour trouver du travail, poussé vers la ville par l'exode rural. Enfin, la vraie vie entre dans l'enseignement des langues. Il est vrai que la mort est toujours absente des manuels, qu'on n'y enterre personne, qu'on n'y souffre même jamais de maladie grave, au pire un rhume passager. Avec la sociolinguistique et l'ethnographie de la communication (Hymes, Goffman, Hall sont, peu après, traduits en français), une logique d'introduction du réel fait surgir un autre monde où vont se produire des interactions, où les personnages, peu à peu, se font acteurs sociaux. ${ }^{13}$

11 Crédif : Centre de recherche et d'études pour la diffusion du français.

12 Javier Suso Lopez, Anales de Filología Francesa, no 1/1, 2002-2003, note 10, http://revistas. um.es/analesff/article/viewFile/19491/18821.

13 Les stéréotypes survivent pourtant bien : récemment encore, je relevais dans un manuel de Corée du Sud vingt-sept images de la Tour Eiffel... 
Il est alors étrange, pour moi, d'éprouver sur le terrain, aux États-Unis, combien cette approche est minoritaire : la DLES dominante à New York m'y apparaît empreinte de linguistique bien plus que d'innovation méthodologique et de recherche de la différence culturelle. Ou du moins, si elle s'intéresse à la figure de l'autre, c'est sous l'angle des stéréotypes, presque sous l'angle de l'exotisme, l'image de la langue française étant celle du bon goût, d'un " chic " que je retrouverai en Asie bien des années plus tard. La passion des intellectuels de l'époque pour la French Theory, la lecture de Roland Barthes, de Jacques Derrida, de Jean-Michel Foucault et le capital culturel français (Langlois vient parler à New Paltz de la Cinémathèque et de la Nouvelle Vague) domine la pratique de la langue elle-même, dans un Département universitaire qui fédère, dans une heureuse ouverture d'esprit, les études de Romance Languages. ${ }^{14}$

Parallèlement, en France, on est passé successivement à une méthodologie audio-visuelle de deuxième génération et, comme au XIX ${ }^{e}$ siècle, où les méthodes de Berlitz étaient nées quand on put sillonner l'Europe en chemin de fer, c'est dans les années 75-80 qu'apparaissent des programmes européens de langues étrangères destinés aux migrants et à la mobilité des travailleurs. La DLES oblige à aller voir ailleurs, se déporter vers d'autres réalités, du côté, du sociétal, de la politique et de l'économie.

\section{Université Paris 3, nommer la didactique}

L'université Paris 3 est alors un foyer passionnant où l'on apprend à nommer tout ce qui constitue la DLES (et, banalement, nommer, c'est toujours dominer). ${ }^{15}$ Quand le monde anglophone, plus pragmatique peut-être, parle de Language Learning and Teaching, nous disons, avec Robert Galisson, "didactologie ", et Swiggers, en Belgique, invente "didaxologie ». L'hyperonyme Didactique, qui combine les moyens, les pratiques et la théorisation qui en est faite, pourra sembler suffisant.

\section{CREDIF - ENS Saint-Cloud, de la recherche au terrain}

Dans ses activités, le Crédif-ENS Saint-Cloud compte une formation longue à l'ingénierie éducative, vouée à préparer à la conception et à la direction de projets et de programmes, parmi lesquels ceux de l'Éducation Nationale et du Ministère des Affaires Étrangères. C'est de cette articulation entre la théorie élaborée dans les écoles doctorales et les séminaires, d'une part, et l'action sur le terrain, d'autre part, que la didactique a besoin

14 James Simpson (éd.), The Handbook of Applied Linguistics, Abingdon. Routledge, 2011. Voir spécialement : Scott Thornbury, "Language Teaching methodology ", p. 185-199.

15 Daniel Coste et Robert Galisson (éds) publient en 1976 un dictionnaire - fondamental - de didactique des langues. 
pour développer une approche qui va envahir rapidement le champ : celle du communicatif. Cependant, il manquerait à cette articulation un cadre conceptuel plus large et c'est ce cadre que développe, autour du professeur Daniel Coste, l'Équipe d'Accueil Plurilinguisme et Apprentissages avec laquelle j'ai pu travailler plusieurs années. Sur le plan théorique, les formes du plurilinguisme individuel y sont étudiées et situées dans leur spécificité, le rôle de l'équipe et particulièrement du professeur Coste s'inscrivant dans la construction d'un plurilinguisme attendu au niveau européen. Associer formation, terrain et recherche me semble un idéal auquel on parvient rarement.

\section{Inalco, langues et cultures distantes en dialogue}

À partir du début des années 80, L'Institut National des Langues et Civilisations Orientales de Paris va être pour moi l'occasion de contribuer, avec une anthropologue et sous l'autorité du Professeur Pierre Vérin (vice-président, professeur de malgache), à la création de la section de français langue étrangère (FLE). L'Institut est un creuset rendu magique par le contexte linguistique et culturel qu'il offre : la possibilité d'une rencontre avec des étudiants de toutes origines, au contact de près de 95 langues, typologiquement souvent si différentes. Ces langues peuvent se répondre et se contredire ainsi dans l'individu, qui est, comme on le sait depuis Uriel Weinreich, le lieu où vit le plurilinguisme, et des cultures distantes entrent ainsi en dialogue avec le français et autour de son apprentissage, en particulier des cultures éducatives, des manières d'être à la langue. Les diplomations de FLE qui venaient d'être mises en place dans les universités françaises (licence, maitrise dont j'étais responsable en parallèle à l'Université d'Orléans) me donnaient l'opportunité d'une réflexion méthodologique que j'allais ensuite amplifier en quittant l'Inalco. ${ }^{16}$

\section{Paris 8, Sciences du langage, Cadre Européen, et recherches doctorales}

À l'université Paris 8, la linguistique s'ouvre à des champs nouveaux et le terme de Sciences du Langage s'impose communément. La composante didactique s'est développée avec E. Companys, puis B. Grandcolas et R. Vivès, les travaux de psycholinguistique avec C. Perdue et la revue Aile (Acquisition et Interaction en Langue Étrangère). Les collaborations avec les réseaux européens se multiplient. J'ai l'occasion de travailler en Europe de l'Est et jusqu'en Sibérie, avec l'université d'Irkoutsk. Mais c'est en sociolinguistique, alors que se diffusent les apports fondateurs de Labov, Haugen, Bernstein et de l'école française de Paris $V$ où j'ai fait ma thèse avec Louis-Jean Calvet, que je reprends contact avec les problématiques africaines, créoles, arabophones et

16 La Professeure Geneviève Zarate allait y créer l'équipe de recherche multilingue Plidam dont j'accompagnerais bien plus tard les travaux. 
celles des aires postcoloniales. Paris 8 est une université-monde où les étudiants apportent autant qu'ils prennent : diriger des thèses pour le Congo, l'Égypte, la Palestine ou le Niger est source d'un élargissement scientifique incomparable.

Puis un peu inopinément, survient en 1998 le Processus de Bologne d'harmonisation des enseignements supérieurs et naît l'ambition européenne du Cadre Européen Commun de Référence pour les Langues (CECRL). Le corpus théorique de la DLES incline rapidement à s'appuyer alors sur une politique européenne des langues qui va lui donner de nouveaux outils et de nouvelles thématiques, et changer partiellement ses objectifs et ses orientations. Les descripteurs, les échelles de compétences, l'approche nouvelle dite " actionnelle ", faisant l'hypothèse que l'apprenant va employer socialement la langue apprise, le concept d'apprenant acteur de sa mobilité ont-ils à ce jour provoqué une véritable révolution ? On en jugera difficilement. La diversité linguistique passe pour devenir un mode d'être à l'Europe multilingue et multiculturelle.

En tout état de cause, la DLES est vue comme une discipline d'intervention sociale. ${ }^{17}$ L'esprit qui est celui de l'université Paris 8 , ancrée au nord de la capitale, va bien dans cette direction. Enseigner, didaskein en grec et in-signare en latin, c'est, très concrètement, signaler, donner du sens, donner un sens. Non pas lire son manuel devant une classe glacée. Faire de la langue une langue vivante. Ces années à Paris 8 (qui n'est pas le seul lieu, bien sûr, où les choses se soient passées ainsi), c'est aussi, avec le Département FLE/Communication, le temps de relire les précurseurs, Carl Rogers, Célestin Freinet, John Dewey, la démarche de projet, les pédagogies dites alternatives qui explorent d'autres voies. Les sciences du langage restent bien présentes, mais l'esprit est ouvert, au delà des territoires académiques. Dans les bureaux, à côté du mien, des collègues de sciences de l'éducation, un psychanalyste... Molière, à la rescousse : Laurent, ne serrez pas ma haire avec ma discipline...

\section{AUF, une diversité francophone}

J'ai vécu à l'Agence Universitaire de la Francophonie (AUF), basée à Montréal et à Paris, un dernier moment de cette ouverture épistémologique vers d'autres horizons. Si le français, à n'en pas douter, tient un rang honorable sur la carte politique des langues du monde, le rôle de l'AUF va bien au delà de la diffusion linguistique. Ce qui me semble le plus fécond encore dans son action d'envergure transnationale est le projet de développer les sciences et les technologies à travers le français et de ne pas considérer la langue comme une fin en soi. Coordonner un réseau francophone international de socio-

17 Pour un point de vue général : Dominique Macaire, Jean-Paul Narcy-Combes et Henri Portine (éds), Interrogations épistémologiques en DDL, Le français dans le monde, Recherches et Applications, $\mathrm{n}^{\circ} 48,2010$. 
linguistique de l'AUF tel que "Dynamique des langues et Francophonie ", Programme "Diversité linguistique et culturelle ", m’a fait rencontrer d'autres pratiques, d'autres manières de concevoir la transmission des langues, et surtout comprendre que la langue ne loge pas dans une case désignée, mais se prête à ouvrir toutes les portes. C'est ainsi que fonctionne depuis deux ans, en Afrique de l'Ouest, une des Écoles doctorales de l'AUF. Elle regroupe chaque année des étudiants de troisième cycle venus de neuf pays différents, spécialistes de langue, de littérature ou de didactique qui, avec notre équipe de six universitaires africains et français, échangent leurs pratiques de recherche et finalisent leurs travaux.

\section{MONDES CRÉOLES. Au delà des disciplines}

Il faut passer à un deuxième temps de notre parcours commun. Celui-ci aidera à prendre conscience de la réalité des frontières, et sans doute de leur intérêt, comme l'a montré Régis Debray, ${ }^{18}$ mais aussi de l'importance qu'il $\mathrm{y}$ a à savoir les dépasser quand elles deviennent des obstacles à la pensée. La notion de discipline reste évidemment de celles-là pour la réflexion en DLES.

On sait comment, à la différence de la Renaissance et l'époque encyclopédique des Lumières, le travail scientifique du $\mathrm{XIX}^{\mathrm{e}}$ et du $\mathrm{XX}^{\mathrm{e}}$ siècles aura consisté en un long effort de circonscription, de bornage du champ de la connaissance, à travers des disciplines distinctes. Nous vivons un autre temps : celui d'un décloisonnement et d'une rupture assumée, avec d'autres approches. Sera ainsi qualifié d'interdisciplinaire un processus d'articulation de plusieurs disciplines intervenant dans l'étude d'un même objet; de pluridisciplinaire une approche de complémentarité dans l'étude d'un objet abordé sous différents points de vue; de transdisciplinaire, une posture épistémologique d'étude d'un même objet dans sa complexité. ${ }^{19} \mathrm{Il}$ y aurait à citer un mot de Goethe sur la question, un mot que rappelle Régis Debray : "L'inconnu se trouve aux frontières des sciences, là où les professeurs se "mangent" entre eux, comme dit Goethe. » Debray ajoute : "Je dis "mangent" mais Goethe n'est pas si poli. ${ }^{20}$ L'idée d'indiscipline va avec ce "dissentiment " dans le savoir que décrivait Lyotard comme un signe historique de la postmodernité. ${ }^{21}$

Aux Antilles, à la Réunion, à l'Ile Maurice, j'ai eu l'occasion de sentir le prix de l'Histoire. J'y ai approché des sociétés plurilingues et pluriculturelles où une réalité post-esclavagiste pesait encore de tout son poids sur les attitudes

18 Régis Debray, Éloge des frontières, Paris, Gallimard, 2010.

19 Je reprends simplement, pour faire bref, la distinction opérée par Patrick Chardenet, in Philippe Blanchet et Patrick Chardenet (éds), Guide pour la recherche en didactique des langues et des cultures, Paris, EAC/AUF, 2014 ( $2^{\mathrm{e}}$ éd.).

20 Régis Debray, op. cit., p. 68.

21 Jean-François Lyotard, La Condition post-moderne, Paris, Éditions de Minuit, 1979. 
et les comportements, fût-ce avec bien des anachronismes : la Martinique département français depuis plus de trente ans, ne pouvait être vue dans les années 80 comme au temps de la Rue Case-Nègres, mais Zobel était étudié dans les classes, le film d'Euzhan Palcy venait de réanimer le texte et le discours du passé, dans un climat politique tendu, était toujours vivant. La situation sociolinguistique était celle d'un Français Langue Seconde qui divisait une société antillaise où l'hypercorrection linguistique était courante. Toute une classe moyenne, solidement éduquée, s'ouvrait à l'anglais du Black Power des États-Unis comme à l'espagnol de Cuba et de José Marti. En pleine diglossie, la minoration linguistique était telle que, dans une de mes enquêtes, certains des répondants oubliaient de mentionner le créole, leur langue maternelle, comme faisant partie de leur répertoire.

Ma thèse, en cours alors, portait sur Saint-Martin, île où la communication sociale se partage entre français, anglais vernaculaire, néerlandais et langues de l'immigration. Elle me donnait à réfléchir à ce que pourraient être les voies d'une éducation multilingue quand le statut politique et le cadre administratif ne s'y prêtent encore guère. Responsable d'un CEFISEM en Guadeloupe $^{22}$, je voyais aussi quels enjeux culturels étaient ceux de la langue de scolarisation que constituait le français et quel était son impact identitaire chez des enfants venus de Dominique, d'Haïti, de Saint-Domingue, arrivant dans des conditions d'intégration difficiles aux Antilles françaises.

Bien plus tard, travaillant en Haïti pour un programme d'évaluation de la coopération régionale, j'ai pu mesurer les enjeux éducatifs et le rôle qui est celui des langues dans le développement. L'école haïtienne vit un bilinguisme français-créole qui a été théorisé par les intellectuels et pris en compte par les politiques, depuis au moins la réforme Bernard voici quarante ans bientôt. Paradoxalement, coexistent une production littéraire brillante (en partie liée à l'émigration de l'élite) et un analphabétisme considérable de la population, avec des "sur-âgés ", des enfants de quatorze ou quinze ans encore à l'école primaire. La situation après le séisme de 2010 ne s'est guère améliorée, semblet-il. Une langue étrangère ou seconde est une condition de l'emploi, parfois de la vie, de la survie.

Mon retour en Haïti m’a fait ressentir tellement de différences avec les Antilles françaises où $\mathrm{j}$ 'avais travaillé presque dix ans, et tellement, bien sûr, avec des îles comme Maurice et la Réunion où la question linguistique prend avant tout un caractère plus identitaire. ${ }^{23}$ Encore une fois, les approches globales, à la fois historique, géolinguistique, ethnologique, économique, sont

22 CEFISEM : structure académique d'appui à la scolarisation des enfants de migrants, aujourd'hui remplacé par les CASNAV.

23 Écart entre la langue de l'école et celle de la famille : pourquoi, à la Réunion, certains enfants sont-ils des "petits-parleurs"? 
nécessaires. Et les choses évoluent vite. Je ne me risquerais pas à parler d'un biotope comme celui de la Guyane, où le multilinguisme a tellement changé de l'immigration surinamienne que j'ai connue à la présence brésilienne d'aujourd'hui et je serais tout autant circonspect, en dépit de la connaissance que j'en ai eue, sur la situation dans l'île de Saint-Martin. ${ }^{24}$

\section{AFRIQUE. L'ingénierie de terrain}

\section{Maghreb, la transition postcoloniale}

Dès le lendemain des indépendances, l'ingénierie éducative va entrer dans la stratégie de développement des pays africains. Elle va les amener à refondre les curriculums en langues et à réviser leurs partenariats d'éducation et de recherche. Dès 1975, j'ai la chance de participer à l'effort de l'Algérie pour se reconstruire. Des voies ont été explorées, parfois lacunaires, parfois fautives, sous la férule des courants idéologico-politiques dominants : l'arabisation à outrance (en littéral, non pas en dialecte), pour laquelle manquent un environnement, des formateurs qualifiés, un esprit (quid des langues berbères ?). La volonté même d'éradiquer la culture de l'ancien colonisateur fait imaginer un enseignement "culture-free ", comme on dirait en anglais. Une circulation humaine et théorique entre la France et l'Algérie montre bien que les fils ne sont pas coupés même si les situations ne sont plus les mêmes et si elles continuent à évoluer. Aux années sombres que va ensuite vivre le pays succédera une embellie. L'école doctorale franco-algérienne en sera un moment fort.

Le cas du Maroc et celui de la Tunisie sont, certes, encore différents, moins heurtés. La scolarisation globale y est meilleure. Une mission d'évaluation des universités met en lumière à mes yeux les disparités des situations, entre la Manouba de Tunis, Sfax ou Sousse et Gabès. La position du français et des langues, l'arabe (les arabes), et l'anglais aussi qui suscite l'engouement d'une jeunesse entreprenante, induisent encore une fois la nécessité de didactiques diversifiées, qui prendraient en compte ces disparités. Or les manuels sont souvent généralistes, rédigés pour des publics irréels ou supposés.

\section{Éthiopie et Djibouti, curriculum et autonomie universitaire}

Si l'on compte les points positifs, le contexte éthiopien est remarquablement favorable à la diffusion du français : il y a, historiquement, la présence religieuse, le chemin de fer qui relie Addis Abeba à Djibouti la francophone, le lycée Guebre Mariam, l'Union Africaine et ses diplomates, le renommé

24 Pierre Martinez, Langues et société aux Antilles. Saint-Martin, Paris, Maisonneuve et Larose, 1994. 
Centre français d'études éthiopiennes, etc. L'extension du Département de français dans une université largement vouée à la langue anglaise, la nomination d'une directrice désormais titulaire d'un doctorat de didactique, la patiente création d'un curriculum adapté aux besoins et aux ambitions, telles devaient être les étapes du développement pour l'AAU. Mais l'Éthiopie, où vécut Arthur Rimbaud, ne s'en tient pas là et ailleurs encore peuvent naître d'autres initiatives, au fil des créations de nouvelles universités dans les régions.

À Djibouti dans les années où j'y ai vécu, un embryon d'université fonctionnait sans autonomie, liée dans une étroite dépendance par des accords de partenariats léonins : polycopiés arrivant par bateau et professeurs français missionnaires et examinateurs en tournées périodiques par avion, qui donnaient des cours intensifs, en droit, en histoire, en lettres, à des étudiants (afars et somalis), plusieurs heures par jour. Surcharge cognitive, chaleur de classes surchauffées, distances rendaient peu efficace un tel dispositif, coûteux aussi pour l'État djiboutien. La décision politique est prise en 2005 de passer à une méthodologie hybride, de renégocier la coopération et de s'appuyer massivement sur les moyens numériques (par ailleurs disponibles dans un excellent centre informatique dédié). À la Faculté de lettres, la politique d'appui en bourses de la coopération française avait, au fil des années, permis la formation en France de plus d'une poignée de docteurs compétents. Ils allaient enfin trouver leur place dans le nouveau dispositif. Aujourd'hui, une ingénierie éducative moderne est au service une Université de plein exercice où le français est vecteur de développement. Une coopération "sud-sud» (selon la formule consacrée) entre l'Éthiopie, Djibouti et les pays de la Mer Rouge a été établie. Il y a là un exemple clair de ce que peut être aussi la DLES.

\section{ASIE. Pluridimension des contextes}

Géopolitique des langues, innovation technologique, approche cognitive : ces questions se posent avec une acuité croissante à l'heure actuelle en Asie sous l'effet de deux facteurs qui jouent à grande échelle. D'une part, pour ce qui est de la géopolitique, ce sont les conséquences qu’a eue la remise en cause des limites des États et des pouvoirs en place hérités d'un ordo mundi de 1945, puis de la décolonisation (Yalta et Bandoeng ayant été des prémices ou des temps forts de cette recomposition) $)^{25}$; et d'autre part, c'est la globalisation des technosciences, et le développement de la communication par internet, qui ne laisseront bientôt aucune zone de la planète à l'écart : toute une partie de l'Asie, comme l'Afrique, d'ailleurs, a sauté l'étape de la cabine téléphonique pour passer directement au smartphone.

25 Voir généralement : Pei-Tseng Jenny Hsieh, Education in East Asia, Londres, Bloomsbury, 2013. 


\section{Inde, la diversité linguistique}

Un projet auquel j'ai été brièvement associé me semble de nature à éclairer la façon dont est abordée aujourd'hui la mise en place d'une diffusion linguistique : le montage d'une formation de Master plurilingue à l'université de New Delhi. L'idée de départ est assez proche de celle qui régit la pensée européenne actuelle en matière de plurilinguisme : il s'agit d'organiser un tronc commun de formation en didactique de deux ans (en anglais) puis de permettre aux futurs enseignants une spécialisation par le jeu d'options (anglais, français, espagnol, allemand). C'est une bonne entrée en matière vers la diversité linguistique et la capacité de la penser ensemble.

\section{Corée, une culture éducative}

Mon expérience coréenne sera plus longue et plus complexe. L'histoire du pays, où j'ai enseigné trois ans à la Faculté d'Éducation de l'Université nationale de Séoul (SNU), reste marquée par l'occupation japonaise, la séparation du nord et du sud, puis, brutalement, l'avènement de la démocratie. Il faut souligner comme y coexistent un fond culturel imprégné de confucianisme et le sentiment d'une accélération de la modernité, une omniprésence de la technologie avec un usage quotidien du numérique. ${ }^{26}$ Cela se traduit, dans l'enseignement des langues, par l'impossibilité presque absolue, pour un étudiant, de poser une question au professeur (car ce serait remettre en cause sa parole ou tenter de se distinguer dans la classe), tandis que, presque dans le même temps, on vérifie qu'on a bien compris et on cherche des compléments sur son smartphone. De même, la méthodologie académique, du moins en sciences humaines, rend difficile la publication d'une pensée qui sorte des sentiers battus. L'évolution n'en est pas moins rapide, l'intérêt pour les langues étrangères, manifeste (par exemple, à l'égard d'un français pour l'Afrique francophone, mais aussi pour le japonais et le chinois... qui attirent de plus en plus). À la SNU, une coopération régionale et une mutualisation scientifique intense se mettent en place avec le Japon, la Chine ou encore Singapour.

\section{Japon, l'homogénéité}

J'ai retrouvé plus tard au Japon cette volonté de voir s'enrichir l'offre de formation en langues étrangères, de développer une culture éducative ouverte, qui rompe avec la compétition entre langues européennes dans des pays où elles ont du mal à se faire une place. En Asie, l'étudiant.e a d'abord un large choix à faire entre les langues asiatiques elles-mêmes avant de songer à apprendre une

26 Pierre Martinez (éd.), Dynamique des langues, plurilinguisme et francophonie. La Corée, Paris, Riveneuve, 2013. 
langue européenne autre que l'anglais, ou plutôt l'anglo-américain. Comme en Corée, ce ne sont pas les moyens de diffusion technologiques qui manquent, mais on observe la persistance d'un conformisme, le poids d'une culture éducative qui a, bien sûr, aussi de grandes qualités, mais s'avère peu propice à l'innovation, avec une place excessive donnée à l'évaluation. Dans cet esprit d'indigénation qui a tant servi le pays depuis Meiji, un grand penseur de la modernité japonaise comme Fukuzawa aurait su, sans doute, imaginer quelque solution originale à ce problème..$^{27}$ Le Japon vit toujours face à des choix difficiles, que n’a pas rendu plus aisés la présence américaine depuis 1945.

\section{Vietnam, l'élargissement}

Il me manquerait de ne pas dire un mot de l'Asie du Sud-Est, et en particulier du Vietnam. Depuis le doi moi de 1989, le pays s'est ouvert sur ses voisins, et un fait marquant me paraît être la coopération éducative qui se déploie entre pays du Mékong, ancienne Indochine et Thaillande. L'action du CREFAP/OIF, celle de l'AUF régionale ${ }^{28}$ (pour ce qui est, encore une fois, d'une langue française qui ne serait plus celle la domination, mais de la coopération et de l'appui au développement) font prendre conscience que des glissements sont possibles, que de nouvelles pistes sont offertes. Sans pouvoir se substituer l'une à l'autre, les médecines occidentales et orientales peuvent se rencontrer. Les cultures éducatives, aussi.

De ces pays où j'ai passé tantôt des mois, tantôt des années, nous devons apprendre la relativité de nos théories et la richesse de la confrontation des idées.

\section{...Ou repenser la DLES}

Comment interpréter ce parcours sinon comme un retour vers le futur? La DLES apparaît comme une praxéologie prête à tous les renoncements s'ils sont raisonnables. Sa résilience au changement m'apparaît indispensable. Il procède d'une bonne dose d'empirisme et non d'une logique prétablie. La didactique se refuse à répéter obstinément, comme la Toinette du Malade Imaginaire : «Le poumon, vous-dis-je, le poumon!».

Une deuxième élément me semble déterminer la constitution scientifique du domaine : c'est une forme d'émergentisme, c'est-à-dire que le tout n'y est pas représenté par l'ensemble des parties mais que le système est, dans son

27 Yukichi Fukuzawa, Plaidoyer pour la modernité, Paris, CNRS, 2008. On lira plus commodément : Pierre-François Souyri, Moderne sans être occidental. Aux origines du Japon d'aujourd'hui, Paris, Gallimard, 2016.

28 CREFAP/OIF : Centre Régional Francophone Asie-Pacifique, Organisation Internationale de la Francophonie, Ho Chi Minh Ville. http://crefap.org/accueils.html ; AUF régionale AsiePacifique, Bureau de Hanoï. https://www.auf.org/asie-pacifique/. 
ensemble, fonction du moment, des conditions, des intrants : ainsi l'apparition du numérique, longtemps retardée par la résistance au changement, par l'inertie assez naturelle des systèmes éducatifs et parfois des hommes, est en passe nolens volens de bouleverser le dispositif de la transmission des langues. C'est pourtant une difficulté, et c'est sans doute plus souvent le cas dans les sciences du corps ou de l'esprit que dans les sciences dites "dures", d'accepter des remises en question qui portent sur des processus et des interactions, sur la relation entre un enseignant et son élève, ou sur les rôles respectifs dévolus à l'homme et à la machine. Cette remise en question, je l'ai posée comme un principe de l'action ; elle affecte le cloisonnement disciplinaire, qui ne tient plus face à l'épreuve du terrain.

Cette réflexivité, cette posture critique inhérente à la DLES a des conséquences sur bien des plans. Je me limiterai à trois d'entre eux.

En matière d'ingénierie éducative, elle contraint à une veille technologique permanente, qui ne peut plus passer par les seuls fonds des bibliothèques et doit s'ouvrir à une pensée globale. Une thèse devrait comporter une sitographie aussi importante que la bibliographie. Une thèse d'un doctorant de tel pays devrait également inclure une biblio-sitographie de sa propre production nationale : quelle forme de néo-colonialisme s'exerce sur l'esprit quand on laisse à croire que rien n'a été pensé, dit ou écrit dans le pays sur lequel d'ailleurs porte souvent la recherche?

En matière de géolinguistique, cette réflexivité doit prendre la mesure des enjeux contemporains. Ainsi, en Asie, la multiplication de campus délocalisés (campus offshore) où l'enseignement, mais aussi toute la vie académique se font en anglo-américain induit une stérilisation de la pensée, fait oublier la notion même de structures mentales et culturelles originales au profit d'un risque de clonage de la pensée, de la recherche et de l'expression.

Enfin, l'esprit critique qui anime la DLES la rend attentive et réceptive à l'innovation contrôlée. Les technosciences, sous l'acronyme des $\mathrm{NBIC}^{29}$, nous guident vers des modes d'apprentissage qui seront ceux de notre siècle. Ce ne sont certes pas le Tableau Blanc Interactif, ni le Powerpoint qui vont révolutionner notre enseignement des langues, mais l'Intelligence artificielle, les robots omniprésents déjà dans tous les secteurs de l'activité humaine, la santé, la politique, les médias, le commerce. C'est une approche plus fine de l'apprenant, dans son profil, ses stratégies, ses spécificités individuelles mais aussi ethnoculturelles qui entre aussi dans cet ordre de réflexion. ${ }^{30}$ C'est la connaissance de la cognition et l'amélioration de nos capacités humaines

29 NBIC : Nano-biotechnologies, Informatique et Communication.

30 Miao Lin-Zucker, Elli Suzuki, Nozomi Takahashi et Pierre Martinez (éds), Compétences d'enseignant à l'épreuve des profils d'apprenant. Vers une ingénierie de formation, Paris, EAC, 2011. 
(cette hominiscence dont parle Michel Serres) ${ }^{31}$, c'est le soldat augmenté, dont la mémoire, la vision, la fatigue sont déjà modifiées, transformées, par les nootropiques, vitamines, molécules de synthèse, puces intradermiques et entraînement : rêve ou cauchemar ? On n'en jugera pas ici. ${ }^{32}$

Voici donc, comme les fantômes de Murnau, qui attendaient Nosferatu de l'autre côté du pont, les vieux démons de la DLES. Regardons-les.

D'abord, une épistémologie "plate", parce qu'elle souffre à mon sens, de ne pas se frotter à la comparaison avec d'autres mondes. Construire une théorie générale imposerait qu’on veuille bien se glisser entre énonciation (conditions de la production, en situation historique et ethnoculturelle) et cognition (dans un processus multifactoriel : âge, genre, biographie, motivation, finalités de l'apprentissage, etc.).

La DLES lutte encore contre la monoculture scientifique qui a fait d'elle un domaine trop longtemps " discipliné ». Heureusement, les choses évoluent, à la faveur de la troisième révolution qui l'affecte, celle des NBIC après la linguistique et les sciences sociales du XXe siècle. Elle semble avoir admis la complexité, qu'elle analyse (décompose) pour être capable de résoudre et d'anticiper.

Enfin, la DLES doit se forcer parfois à accepter l'innovation, et l'éducation en langues n'échappe pas aux résistances qu’avait dévoilées, voici longtemps, Everett Rogers. Comme une économie de la connaissance ne suffit pas, il faut savoir, interpréter et diffuser. ${ }^{33}$ Il resterait à évaluer dans quelle mesure le présent moment de la vie de la DLES correspond à une de ces phases du paradigme que Thomas Kuhn appelle la "science extraordinaire ", où la recomposition du paradigme en crise est en train de s'opérer. ${ }^{34} \mathrm{Je}$ le crois personnellement, parce qu'il ne me semble pas envisageable que soit prolongé longtemps le modèle épistémologique que nous avons connu. On peut bien sûr penser autrement. J'appelle cela se mettre la tête dans le sable.

J'en viens à l'épilogue d'un parcours. De retour à Dante et à son goût pour le doute plus que pour le savoir, ce parcours amène à se poser des questions, qui sont autant de défis :

1) Quelle est la valeur actuelle de notre éducation en langue étrangère ? Qu'apporte-t-elle ? Permet-elle d'entendre l'altérité, de rencontrer l'autre ? Quel doit être le rôle des éducateurs médiateurs de cette rencontre dans les

31 Michel Serres, Petite Poucette, Paris, Le Pommier, 2013. Michel Serres, Le gaucher boiteux. Figures de la pensée, Paris, Le Pommier, 2015.

32 Éric Sadin, L’humanité augmentée, Paris, L'Échappée, 2013. Séminaire militaire, Paris, 19 juin 2017 : https://iatranshumanisme.com/2017/06/14/seminaire-sur-le-soldat-augmente/.

33 Everett M. Rogers, Diffusion of Innovations, NYC, Free Press, 1962, 2003. Ouvrage recensé dans Essais 1/I, 2012. Pierre-Yves Citton, L'avenir des humanités. Économie de la connaissance ou cultures de l'interprétation?, Paris, La Découverte, 2010.

34 Thomas Kuhn, La structure des révolutions scientifiques, Paris, Champs Flammarion, 1962, éd. fr., 1983. 
nouveaux environnements d'apprentissage ? Et si l'on admet l'idée d'une science participative ou collaborative, ${ }^{35}$ qu' est-ce que les étudiants (" bénéficiaires ", usagers, acteurs) ont à en dire ? Une des questions les épineuses est que, contrairement à bien d'autres domaines scientifiques, en sciences dites "dures " mais même en sciences humaines qui le seraient moins, la validation expérimentale est difficile, voire parfois impossible. Comment évaluer la pertinence qu'aura eue une démarche pédagogique et didactique sur le plan des compétences actionnelles à long terme ou sur son impact dans la transformation culturelle ou identitaire chez un apprenant (et pire encore chez un grand nombre d'apprenants) ? La validation expérimentale d'une mesure, d'une méthode ou d'un programme, après coup, ex post facto, ${ }^{36}$ est le fondement d'une scientificité. Elle doit s'imposer à nous et nous devons apprendre aux jeunes chercheurs à en faire le trébuchet de leurs travaux.

2) Quelles finalités pouvons-nous assigner à l'apprentissage des langues au $\mathrm{XXI}^{e}$ siècle? Allons-nous adopter des objectifs instrumentaux (voyager, travailler), culturels ? Philosophiques (par exemple la recherche d'une humanité ou d'une identité) ? En tout état de cause, l'éducation en langues constitue un dispositif social avec ses conséquences sur l'humain, dont on ne saurait faire l'économie. ${ }^{37}$

3) Quelles transitions, à partir des nouvelles conditions de l'apprentissage, vers une autre didactique et comment opérer ces transitions ? Concrètement, s'imposent trois contraintes : une connaissance systémique des environnements de cet apprentissage (souvent hybride ou nomade) ; une exploitation intégrative des ressources (par exemple, ne pas limiter le numérique à une fonction ancillaire) ; enfin, à travers le curriculum, l'inclusion fonctionnelle des acquis de l'expérience et de l'innovation pour une organisation rationnelle des moyens.

4) Enfin doit-on se demander ce que peut être un chercheur en didactique des langues, et la question passe par une réflexion sur le positionnement (institutionnel) et sur la posture (professionnelle) de celui-ci, entre objectivation et implication. Il est clair qu'une recherche totalement impliquée relève du militantisme, qu'elle est ascientifique, et il peut arriver qu'on ait à dire à un doctorant qu'il faut savoir établir un rapport ou une proportion entre expérience et/ ou réflexivité. Dans ce registre, qui confine à l'éthique, se pose la question du local (ce qui est possible) et du global (ce qui est non pertinent). Le chercheur doit être conscient qu'il est investi d'une responsabilité, parce que les résultats de son travail sont attendus et, s'ils en valent la peine, seront entendus.

35 Rapport François Houllier, Les sciences participatives en France, MEN, 2016.

36 Ex post facto. Voir Louis Cohen et al., Research Methods in Education, London, Routledge, $7^{\text {th }}$ Ed., 2011, p. 303.

37 La tentation est grande pour certains pays, en Asie, en Europe même, de rendre facultative l'étude, fût-ce d'une seule langue étrangère dans le cursus. 
Les nouveaux défis traînent à leur suite, on le voit, bien des préalables à l'intervention didactique. Je tirerais de ce programme de travail, qui peut sembler un peu de l'ordre de l'incantation, un principe dominant : il doit conduire à développer et articuler toujours plus efficacement la recherche universitaire, la décision politique et l'action éducative.

Au delà de ces défis - la valeur, les finalités, la transition, la recherche - il est périlleux de dessiner l'avenir. S'il fallait se risquer à pointer du doigt un dénominateur commun de la pensée occidentale contemporaine en matière d'éducation en langues, ce serait, à mon sens, du côté de la construction de l'individu, de la personne, qu'on pourrait chercher. L'hypothèse me semble pouvoir être étayée par notre histoire culturelle, la romanitas, qui ne se fonde pas sur une seule langue, la diversité politique de la France du Traité de Verdun à l'abbé Grégoire, le goût pour le plurilinguisme, avec latin, français, angloaméricain successivement érigés en lingua franca, au fil du temps, etc. D'autres visions humanistes de l'étude des langues étrangères peuvent se rapprocher de celle-ci à certains égards, mais s'en détachent profondément à d'autres : traditions de la medersa arabo-islamique, force de l'écrit qui fait le lettré dans le monde des sinogrammes, esprit confucéen jouant sur les hiérarchies et les apprentissages. Il me semble avoir côtoyé ces différences, mesuré ces écarts, un peu compris ces différences.

Parce que l'horizon d'attente de ma réflexion, on l'aura compris, est orienté non par une méthodologie qui serait la panacée (éternellement) espérée, mais par une philosophie de l'éducation en langues, l'hypothèse de travail que je soumets pour la DLES est celle d'une didactique organisée en réseaux. J'ai, de ce point de vue, le sentiment que les propositions d'un physicien mais aussi un grand penseur que fut Werner Heisenberg, peuvent nous aider à y voir clair. ${ }^{38}$ Cette philosophie fonctionnerait sur la base de réseaux de réalité analysés comme système. Tel qu'Heisenberg le représente, le monde n'est pas fait de choses "matérielles " mais seulement de connexions nomologiques, pas de substances, mais d'objets en relation. Seul l'agencement de ces objets (les moyens, les concepts) permet de construire une représentation du réel et cette façon de penser la DLES dans une approche connexionniste, je l'appelle didactique réticulaire.

Elle renvoie aussi à ce que dit Antoine Compagnon de la théorie, qui ne doit pas être un système de réponses mais un cadre de questions. ${ }^{39} \mathrm{Si}$ ce texte peut donc ouvrir sur quelque conclusion, c'est à une constante restructuration de notre domaine qu'il invite et non à une théorie toute faite. Cette DLES en mouvement, donnant sens et réalité à ses moyens et à ses concepts en les

38 Werner Heisenberg, Le manuscrit de Nuremberg, 1942, rééd. Paris, Allia, 2010, p. 13-14.

39 Antoine Compagnon, Une question de discipline. Entretiens avec Jean-Baptiste Amadieu, Paris, Flammarion, 2013, p. 121. 
mettant en réseaux, s'appuierait fondamentalement aujourd'hui sur le numérique, les neurosciences et l'architecture curriculaire. Elle ne saurait se satisfaire d'ajustements au coup par coup, comme nous y sommes trop souvent invités, en fonction des innovations qui arrivent sans perspective intégrative et qui poussent à l'éclectisme méthodologique désordonné, ou à l'asservissement à une seule finalité primaire de l'éducation en langues (par exemple, son utilité). Elle relève d'une conception de l'homme et de son éducation.

Pierre Martinez

Professeur émérite Université Paris 8 - Saint-Denis

Sciences du Langage et Didactique des Langues pierre.martinez@univ-paris8.fr http://site-pierrealainmartinez.strikingly.com/

\title{
Résumé
}

Ce texte met un trajet personnel, professionnel et scientifique, en regard avec celui qu'a connu depuis plusieurs décennies la Didactique des Langues Étrangères et Secondes. Dans un même mouvement, des points de vue - épistémologie, disciplines, ingénierie, contextes - et des horizons linguistiques et culturels de nature à les éclairer sont présentés. Trois révolutions ont impacté le domaine : sciences du langage, sciences humaines et sociales, technosciences cognitives et informatiques. Mon hypothèse actuelle est que seule une didactique de mise en réseaux ("réticulaire »), appuyée sur le numérique, peut structurer une éducation en langues intégrative et cohérente.

\section{Mots-clés}

Langues étrangères et secondes, paradigme, didactique réticulaire, éducation en langues.

\begin{abstract}
This text matches a personal, professional and scientific path with that experienced for several decades in the Teaching of Foreign and Second Languages. In a same movement, points of view -epistemology, disciplines, engineering, contexts- and linguistic and cultural horizons that can enlighten them are presented. Three revolutions impacted the field: language sciences, human and social sciences, cognitive and computer technosciences. My hypothesis is that only a didactic of networking ("reticular"), supported by digital means, seems now able to structure an integrative and coherent TFSL.
\end{abstract}

\section{Keywords}

Foreign and second languages, paradigm, reticular didactics, language education. 